\title{
EVOLUTION OF MOLECULAR ABUNDANCE IN PROTOPLANETARY DISKS
}

\author{
YURI AIKAWA 円 \\ Department of Earth and Planetary Science, University of Tokyo, \\ Bunkyo-ku, Tokyo 113, Japan \\ TOYOHARU UMEBAYASHI \\ Data Processing Center, Yamagata University, Yamagata 990, Japan \\ TAKENORI NAKANO \\ Nobeyama Radio Observatory, National Astronomical Observatory, \\ Nobeyama, Minamisaku, Nagano 384-13, Japan \\ AND \\ Shoken M. Miyama \\ National Astronomical Observatory, Mitaka, Tokyo 181, Japan
}

\begin{abstract}
We investigate the evolution of molecular abundance in quiescent protoplanetary disks which are presumed to be around weak-line T Tauri stars. In the region of surface density less than $10^{2} \mathrm{~g} \mathrm{~cm}^{-2}$ (distance from the star $\gtrsim 10 \mathrm{AU}$ in the minimum-mass solar nebula), cosmic rays are barely attenuated even in the midplane of the disk and produce chemically active ions such as $\mathrm{He}^{+}$and $\mathrm{H}_{3}^{+}$. Through reactions with these ions $\mathrm{CO}$ and $\mathrm{N}_{2}$ are finally transformed into $\mathrm{CO}_{2}, \mathrm{NH}_{3}$, and $\mathrm{HCN}$. In the region where the temperature is low enough for these products to freeze onto grains, considerable amount of carbon and nitrogen is locked up in the ice mantle and is depleted from the gas phase in a time scale $\lesssim 3 \times 10^{6}$ yr. Oxidized $\left(\mathrm{CO}_{2}\right)$ ice and reduced $\left(\mathrm{NH}_{3}\right.$ and hydrocarbon) ice naturally coexist in this part of the disk. The molecular abundance both in the gas phase and in ice mantle varies significantly with the distance from the central star.
\end{abstract}

Subject headings: circumstellar matter - ISM: molecules — stars: formation — stars: pre-main sequence — solar system: formation

${ }^{1}$ present address: National Astronomical Observatory, Mitaka, Tokyo 181, Japan 


\section{INTRODUCTION}

Radio, infrared, and optical observations have recently revealed the existence of circumstellar disks around young stellar objects (Sargent \& Beckwith 1994; O'dell \& Wen Zheng 1994). Planet formation must be going on at least in some of them. Theoretical study on the evolution of molecular abundance in such " protoplanetary" disks is important from various points of view. First, it will directly show what material the bodies in the planetary systems are made from. Secondly, molecular abundance can be a useful probe in investigating the formation processes of planetary systems. For example, we may be able to reduce the processes, places, and epochs of the formation of primitive bodies, such as comets, by comparing their molecular composition with the theoretical results on the distribution and evolution of molecular abundance in the disk. Thirdly, study of the structure and evolution of the disk by observations of molecular lines should ask for a help of theoretical study on molecular evolution. For example, the amount of the gaseous component in the disk is estimated by observations of molecules other than the main component $\mathrm{H}_{2}$ because $\mathrm{H}_{2}$ can hardly emit photons except at very inner region of the disk. Knowledge on the abundance of the molecules relative to hydrogen is indispensable for such study.

Some theoretical works have been done on the molecular abundance in protoplanetary disks. Prinn and his colleagues (Prinn 1993, and references therein) investigated the molecular evolution in fully turbulent accretion disks and proposed the kinetic inhibition (KI) model. Their basic idea is that the molecular abundance in the matter flowing outwards is " quenched "when its temperature has decreased to a value below which the time scale for chemical reaction is larger than the dynamical time scale. Adopting the lifetime of the disk $\sim 10^{13} \mathrm{~s}$, they concluded that the molecular abundance was quenched at $850-1500 \mathrm{~K}$, and that $\mathrm{CO}$ and $\mathrm{N}_{2}$ were the dominant component in the solar nebula. They considered neutral-neutral reactions which can be efficient in the region of $T \gtrsim$ several $\times 10^{2} \mathrm{~K}$.

In this letter we investigate the evolution of molecular abundance in a relatively quiescent disk in the post-accretion phase, the so-called weak-line $\mathrm{T}$ Tauri (WTTS) phase and thereafter, in which transport and mixing of matter is not so efficient as in the earlier active phases mentioned above. We consider the disk regions where the column density is lower than the attenuation length of cosmic rays, $96 \mathrm{~g} \mathrm{~cm}^{-2}$ (Umebayashi \& Nakano 1981). The minimum-mass solar nebula (Hayashi 1981) satisfies this condition in the region more than about $7 \mathrm{AU}$ away from the central star with the temperature $T \lesssim 10^{2} \mathrm{~K}$. We take into account the ionization by cosmic rays and the subsequent ion-molecule reactions, which were not considered in the previous works.

\section{DISK MODEL AND REACTION NET- WORK}

As the model of the protoplanetary disk, we adopt the so-called minimum-mass solar nebula (Hayashi 1981), which has the the distribution of the surface density $\Sigma(R)=54(R / 10 \mathrm{AU})^{-3 / 2} \mathrm{~g} \mathrm{~cm}^{-2}$ and temperature $T(R)=89(R / 10 \mathrm{AU})^{-1 / 2} \mathrm{~K}$, where $R$ is the distance from the central star. This model is consistent with the distribution of the temperature and the surface density estimated from the observations of dust continuum (Beckwith et al. 1990). Hydrostatic equilibrium determines the density distribution perpendicular to the midplane of the disk (e.g., Aikawa et al. 1996).

We consider the gas-phase reactions, adsorption onto grains, and thermal desorption from grains. The reaction network we use is essentially the same as the one described in Aikawa et al. (1996). We adopt the UMIST94 data base (Millar et al. 1991; Farquhar \& Millar 1993) for the gas-phase reactions, and also take into account some three-body reactions referring to Brasseur \& Solomon (1986). Table 1 shows all the atoms and molecules included in our network (except $\mathrm{H}, \mathrm{H}_{2}$, and $\mathrm{He}$ ) and their adsorption energies. Because the attenuation of cosmic rays is inefficient, we adopt the ionization rate in the dark cloud, $\zeta \approx 10^{-17}$ $\mathrm{s}^{-1}$. We neglect the ionization and dissociation by the interstellar and stellar ultraviolet radiation since it can be attenuated by grains in a thin surface layer of the disk (see $\S 4.3$ ). We take the sticking probability $S=0.3$ for the collision of a gas particle with a grain (Williams 1993). For simplicity, we assume that all grains are spherical with radius $a=10^{-5} \mathrm{~cm}$ and that the dust-gas ratio is the same as that in the interstellar clouds. Although the dust-gas ratio near the midplane might be enhanced by sedimentation of dust, it does not affect much the results; we have obtained almost the same results with the dust-gas ratio higher by orders of magnitude. 
Since our reaction network consists of one- and two-body reactions and contains only a small number of three-body reactions, we consider the region of the density by number of hydrogen nuclei, $n_{\mathrm{H}} \lesssim$ $10^{12} \mathrm{~cm}^{-3}$, which corresponds to $R \gtrsim 10$ AU for the minimum-mass solar nebula. With a typical rate coefficient $k \sim 10^{-30} \mathrm{~cm}^{6} \mathrm{~s}^{-1}$ for the three-body reactions we have in this density range $k n\left(\mathrm{H}_{2}\right) \lesssim 10^{-18} \mathrm{~cm}^{3} \mathrm{~s}^{-1}$ even for the most abundant species $\mathrm{H}_{2}$ as a third body. This is smaller than the characteristic rate coefficient $\sim 10^{-17} \mathrm{~cm}^{3} \mathrm{~s}^{-1}$ for radiative association, which is the slowest among the two-body reactions. Thus the three-body reactions are not important in this density range.

For simplicity and clarity, we assume that the density and temperature do not change with time. Since we are mainly interested in the quiescent (WTTS) phase, we neglect the radial transport of matter and perform calculation up to $\sim 10^{7}$ yr. The initial molecular abundance is the same as in Aikawa et al. (1996), which assumed mostly the observed abundance in the dark cloud TMC-1. However, the molecular abundance may change during the active phase of the disk. To find out the influence of the initial condition, we also perform calculation with another set of the initial abundance; all carbon is in the form of $\mathrm{CO}$, the remaining oxygen is locked up in water ice mantle, and nitrogen is in $\mathrm{N}_{2}$.

\section{NUMERICAL RESULTS}

Since the result is found to be much more sensitive to the temperature than to the density, we show as the representative cases the results at $T=30$ and $90 \mathrm{~K}$ corresponding to the regions of $R=87$ and $9.7 \mathrm{AU}$ with the density $n_{\mathrm{H}}$ at the midplane $2.9 \times 10^{9}$ and $1.2 \times 10^{12} \mathrm{~cm}^{-3}$, respectively.

Figures $1 a$ and $1 b$ show the results for the first set of the initial condition. The solid lines show the time variation of the abundance of carbon-bearing molecules relative to hydrogen. In the region of $T=$ 30K (Figure $1 a$ ) the abundance of CO remains nearly constant at its initial value $n(\mathrm{CO}) / n_{\mathrm{H}} \approx 7 \times 10^{-5}$ up to $10^{6}$ yr. Thereafter $\mathrm{CO}$ decreases steeply, and $\mathrm{CO}_{2}$ ice and $\mathrm{HCN}$ ice become dominant. The main process of forming $\mathrm{CO}_{2}$ and $\mathrm{HCN}$ is as follows. Through the reaction with $\mathrm{H}_{3}^{+}$, $\mathrm{CO}$ is transformed into $\mathrm{HCO}^{+}$, some of which become $\mathrm{HCO}$ mainly through the grain surface recombination. Reactions of $\mathrm{HCO}$ with $\mathrm{C}$ and $\mathrm{N}$ finally form $\mathrm{CO}_{2}$ and $\mathrm{HCN}$ in the gas phase ${ }^{2}$. Once $\mathrm{CO}_{2}$ and $\mathrm{HCN}$ are formed, they are adsorbed by grains in a time as short as $\sim 10 \mathrm{yr}$ (Aikawa et al. 1996). Since these molecules are hardly desorbed at $T=30 \mathrm{~K}$, they are locked up in the ice mantle, and as a result carbon is depleted from the gas phase. At somewhat higher temperatures (e.g., $T=90 \mathrm{~K}$ as in Figure $1 b$ ), the thermal desorption is efficient enough to compensate the adsorption; although $\mathrm{CO}_{2}$ becomes abundant, $\mathrm{CO}$ is also abundant on a time scale of $10^{7}$ yr.

The dotted lines in Figures $1 a$ and $1 b$ show the time variation of the abundance of oxygen-bearing molecules. Oxygen is initially assumed to be mostly in the form of $\mathrm{O}$ atom and $\mathrm{CO}$ molecule. The abundance of $\mathrm{O}_{2}$ in the gas phase and $\mathrm{H}_{2} \mathrm{O}$ ice in the grain mantle increases extensively with time. The $\mathrm{H}_{2} \mathrm{O}$ molecules form mainly in the following process. The reaction between $\mathrm{O}$ and $\mathrm{H}_{3}^{+}$produces $\mathrm{OH}^{+}$, most of which are transformed into $\mathrm{H}_{3} \mathrm{O}^{+}$through $\mathrm{H}$ atomabstractions from $\mathrm{H}_{2}$. Then $\mathrm{H}_{3} \mathrm{O}^{+}$recombines to produce $\mathrm{H}_{2} \mathrm{O}$. In the later stages where $\mathrm{O}_{2}$ becomes dominant, the main reaction of forming $\mathrm{OH}^{+}$is the $\mathrm{H}$ atom-abstraction by $\mathrm{O}^{+}$, which is produced via destruction of $\mathrm{O}_{2}$ by $\mathrm{He}^{+}$. Since $\mathrm{H}_{2} \mathrm{O}$ freezes onto grains at these temperatures while $\mathrm{O}_{2}$ does not, most oxygen freezes out in the form of $\mathrm{H}_{2} \mathrm{O}$ ice and the abundance of $\mathrm{O}_{2}$ decreases in a time scale of several $\times 10^{6} \mathrm{yr}$.

The dashed lines in Figure 1 show the time variation of the abundance of nitrogen-bearing molecules. Initially most nitrogen is assumed to be in the form of $\mathrm{N}$ atoms. As time goes on, $\mathrm{N}_{2}, \mathrm{HCN}$ ice, and $\mathrm{NH}_{3}$ ice become dominant. The main formation process of $\mathrm{NH}_{3}$ is as follows. The reaction of $\mathrm{N}_{2}$ with $\mathrm{He}^{+}$ forms $\mathrm{N}^{+}$, which is transformed into $\mathrm{NH}_{4}^{+}$by repeating $\mathrm{H}$ atom-abstraction. Finally $\mathrm{NH}_{4}^{+}$is turned into $\mathrm{NH}_{3}$ through dissociative recombination and grainsurface recombination. At temperatures below about $70 \mathrm{~K}, \mathrm{NH}_{3}$ freezes onto grains and most nitrogen is depleted from the gas phase. At higher temperatures as in Figure $1 b, \mathrm{NH}_{3}$ cannot freeze and $\mathrm{N}_{2}$ is dominant even at later stages.

\section{DISCUSSION}

\footnotetext{
${ }^{2} \mathrm{By}$ recombination on grains $\mathrm{HCO}^{+}$is transformed into $\mathrm{HCO}$ radical or $\mathrm{CO}+\mathrm{H}$ with unknown branching ratio. We assumed that the former branch is dominant. The time scale of the formation of $\mathrm{CO}_{2}$ and $\mathrm{HCN}$ becomes longer if we assume otherwise.
} 


\subsection{Implications to Planetary Science}

We review the main points of our results and discuss the implications to planetary science. First, we have found that $\mathrm{CO}$ and $\mathrm{N}_{2}$ are transformed into $\mathrm{CO}_{2}$ and $\mathrm{NH}_{3}$. The main cause for this is in cosmic rays, which have not been taken into account in the previous works. Cosmic rays produce chemically active ions such as $\mathrm{H}_{3}^{+}$and $\mathrm{He}^{+}$which can destroy $\mathrm{CO}$ and $\mathrm{N}_{2}$. This results in the depletion of $\mathrm{CO}$ in the gas phase and hence the faintness of its emission lines in the cold regions $(T \lesssim 70 \mathrm{~K})$ of the disks at the age $\gtrsim$ several $\times 10^{6} \mathrm{yr}$. This also means that $\mathrm{CO}_{2}$ ice and $\mathrm{NH}_{3}$ ice will be detected in these regions. Secondly, our results show that oxidized $\left(\mathrm{CO}_{2}\right)$ ice and reduced $\left(\mathrm{NH}_{3}\right.$ and hydrocarbon) ice coexist in the protoplanetary disks. This coexistence has also been suggested by the KI model (Prinn 1993). This result is to be noted because their coexistence is one of the most important characteristics of comets (Yamamoto 1991). $\mathrm{CO}$ and $\mathrm{N}_{2}$ would also coexist in ice mantle if they are physically trapped in water ice (Bar-Nun et al. 1985), although this effect is not included in our calculation. Finally, we have found that the molecular abundance in the gas and in ice mantle varies considerably with the distance from the central star. Comparison of this result with the molecular abundance of comets will enable us to deduce where comets formed, although quantitative discussion is out of the scope of this paper.

\subsection{Dependence on the Initial Condition}

The initial condition we have adopted so far is that considerable amount of carbon, oxygen, and nitrogen are atomic (see $\S 2$ in Aikawa et al. 1996). In order to check the dependence on the initial condition, we have made calculation for another set of the initial abundance mentioned in $\S 2$. Figure 2 shows the result for this case in the region of $T=30 \mathrm{~K}$. We can see that $\mathrm{CO}_{2}$ ice becomes dominant in this case as well. Although there are no oxygen atoms initially, they are extracted from $\mathrm{CO}$ by the reaction with $\mathrm{He}^{+}$. The remaining carbon is transformed into hydrocarbon. At higher temperatures $(T \gtrsim 70 \mathrm{~K})$, $\mathrm{CO}_{2}$ becomes abundant at later stages, although its abundance $n_{\mathrm{CO} 2} / n_{\mathrm{H}}=10^{-5}-10^{-6}$ is lower than that for the first case on the initial condition. Similarly, $\mathrm{N}_{2}$ is destroyed by $\mathrm{He}^{+}$and is transformed into $\mathrm{HCN}$ ice and $\mathrm{NH}_{3}$ ice.

\subsection{Discussion on the Assumptions}

Although we have neglected the radial transport of matter, our results are qualitatively applicable to the disks with radial transport such as the accretion disk and weakly-turbulent disk, because the chemical paths described in $\S 3$ are always active and dominant in the reaction network as long as the matter is in the region of low temperature and low density ( $R \gtrsim 10 \mathrm{AU}$ in the minimum-mass solar nebula). The molecular evolution in the accretion disks will be described in the forthcoming paper in more detail. On the other hand, our results are not applicable to the disks with efficient mixing where the strong turbulence or convection mixes matter between the inner hot $(T \gtrsim$ several $\times 100 \mathrm{~K})$ region and the outer cold region $(R \gtrsim 10 \mathrm{AU})$ in a time scale $\lesssim 10^{6} \mathrm{yr}$, as investigated by Prinn (1993).

We have neglected the UV photolysis. Smaller grains sink more slowly toward the midplane of the disk (Nakagawa, Nakazawa, \& Hayashi 1981). In addition, even a weak turbulence which cannot efficiently transport matter radially would be sufficient to prevent the sedimentation of small grains. Therefore, it would be possible that small grains shield the main part of the disk from the UV radiation. Midto far-infrared excesses observed in the spectra of WTTS strongly suggest the existence of small grains at $R \gtrsim 10 \mathrm{AU}$ even in the relatively quiescent disks (Strom et al. 1989).

If almost all grains sank to the midplane in less than about $10^{6} \mathrm{yr}$ despite the above discussion and the mid- to far-infrared excesses in WTTS, our results were not applicable. The molecular abundance must then be affected by the photolysis because the line shielding is not efficient enough for some kinds of species.

We would like to thank Drs. T. Yamamoto, Y. Abe, and J. Watanabe for helpful discussion. Anonymous referee's comments were useful in improving the manuscript. The numerical calculations were performed partly at the Astronomical Data Analysis Center of National Astronomical Observatory, Japan. This work is supported by Grant-in-Aid for Scientific Research (08640319) of the Ministry of Education, Science, Sports, and Culture, Japan. Y. A. would like to acknowledge support by JSPS Research Fellowships for Young Scientists. 
TABLE 1

THE ADSORPTION ENERGIES OF ATOMS AND MOLECULES IN KELVIN a

\begin{tabular}{|c|c|c|c|}
\hline Species & $\begin{array}{l}\text { Adsorption } \\
\text { Energy }(\mathrm{K})\end{array}$ & Species & $\begin{array}{l}\text { Adsorption } \\
\text { Energy (K) }\end{array}$ \\
\hline $\mathrm{C}$ & 800 & $\mathrm{C}_{2} \mathrm{H}$ & 1460 \\
\hline $\mathrm{N}$ & 800 & $\mathrm{C}_{3}$ & 2010 \\
\hline $\mathrm{O}$ & 800 & $\mathrm{CCN}$ & 2010 \\
\hline $\mathrm{Na}$ & 11800 & $\mathrm{CO}_{2}$ & $2690^{\mathrm{b}}$ \\
\hline $\mathrm{Mg}$ & 5300 & $\mathrm{NH}_{2}$ & 860 \\
\hline $\mathrm{Si}$ & 2700 & $\mathrm{NO}_{2}$ & 2520 \\
\hline S & 1100 & $\mathrm{OCN}$ & 2010 \\
\hline $\mathrm{Fe}$ & 4200 & OCS & 3000 \\
\hline HS & 1500 & $\mathrm{O}_{2} \mathrm{H}$ & 1510 \\
\hline $\mathrm{CH}$ & 650 & $\mathrm{O}_{3}$ & 2520 \\
\hline $\mathrm{C}_{2}$ & 1210 & $\mathrm{SO}_{2}$ & $3460^{\mathrm{b}}$ \\
\hline $\mathrm{CN}$ & 1510 & $\mathrm{H}_{2} \mathrm{CO}$ & 1760 \\
\hline $\mathrm{CO}$ & $960^{\mathrm{b}}$ & $\mathrm{H}_{2} \mathrm{CS}$ & 2250 \\
\hline CS & 2000 & $\mathrm{CH}_{3}$ & 1160 \\
\hline $\mathrm{NH}$ & 600 & $\mathrm{C}_{2} \mathrm{H}_{2}$ & $2400^{\mathrm{c}}$ \\
\hline $\mathrm{N}_{2}$ & $710^{\mathrm{c}}$ & $\mathrm{C}_{3} \mathrm{H}$ & 2270 \\
\hline $\mathrm{NO}$ & 1210 & $\mathrm{C}_{3} \mathrm{~N}$ & 2720 \\
\hline NS & 2000 & $\mathrm{NH}_{3}$ & $3080^{\mathrm{b}}$ \\
\hline $\mathrm{OH}$ & 1260 & $\mathrm{H}_{2} \mathrm{C}_{3}$ & 2110 \\
\hline $\mathrm{O}_{2}$ & 1210 & $\mathrm{HC}_{3} \mathrm{~N}$ & 2970 \\
\hline $\mathrm{SiH}$ & 2940 & $\mathrm{CH}_{4}$ & $1080^{\mathrm{c}}$ \\
\hline $\mathrm{SiC}$ & 3500 & $\mathrm{CH}_{3} \mathrm{O}$ & 1710 \\
\hline $\mathrm{SiO}$ & 3500 & $\mathrm{C}_{3} \mathrm{H}_{2}$ & 2110 \\
\hline $\mathrm{SiS}$ & 3800 & $\mathrm{CH}_{3} \mathrm{CN}$ & 2270 \\
\hline SO & 2000 & $\mathrm{CH}_{3} \mathrm{OH}$ & $4240^{\mathrm{b}}$ \\
\hline $\mathrm{H}_{2} \mathrm{O}$ & $4820^{\mathrm{b}}$ & $\mathrm{CH}_{3} \mathrm{O}_{2}$ & 2370 \\
\hline $\mathrm{H}_{2} \mathrm{~S}$ & 1800 & $\mathrm{C}_{3} \mathrm{H}_{3}$ & 2220 \\
\hline $\mathrm{HCN}$ & $4170^{\mathrm{c}}$ & $\mathrm{H}_{3} \mathrm{C}_{3} \mathrm{~N}$ & 3270 \\
\hline $\mathrm{HCO}$ & 1510 & $\mathrm{C}_{3} \mathrm{H}_{4}$ & 2470 \\
\hline $\mathrm{CH}_{2}$ & 960 & $\mathrm{CH}_{3} \mathrm{OOH}$ & 2620 \\
\hline
\end{tabular}

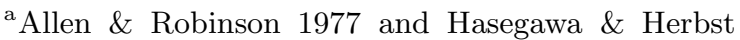
1993 except for ${ }^{b}$ and ${ }^{c}$

${ }^{\text {b }}$ Sandford \& Allamandola 1993

${ }^{\text {c}}$ Yamamoto, Nakagawa, \& Fukui 1983 
Fig. 1. - Evolution of the molecular abundance in two representative regions of the disk, (a) $R=87 \mathrm{AU}$ $\left(n_{\mathrm{H}}=2.9 \times 10^{9} \mathrm{~cm}^{-3}, T=30 \mathrm{~K}\right)$ and $(\mathrm{b}) R=9.7 \mathrm{AU}$ $\left(n_{\mathrm{H}}=1.2 \times 10^{12} \mathrm{~cm}^{-3}, T=90 \mathrm{~K}\right)$. The initial molecular abundance was determined referring to the abundance in dark clouds. The solid, dotted, and dashed lines represent the abundance of carbon-, oxygen-, and nitrogen-bearing molecules, respectively, relative to hydrogen.

Fig. 2.- Evolution of the molecular abundance at $R=87 \mathrm{AU}\left(n_{\mathrm{H}}=2.9 \times 10^{9} \mathrm{~cm}^{-3}, T=30 \mathrm{~K}\right)$ with the initial condition that carbon is in the form of $\mathrm{CO}$, nitrogen is in the form of $\mathrm{N}_{2}$, and the remaining oxygen is locked up in water ice mantle. The other details are the same as in Fig. 1

\section{REFERENCES}

Aikawa, Y, Miyama, S. M., Nakano, T., \& Umebayashi, T. 1996, ApJ, 467, 684

Allen, M., \& Robinson, G. W. 1977, ApJ, 212, 396

Bar-Nun, A., Hertman, G., Laufer, D., \& Rappaport, M. L. 1985, ICARUS, 63, 317

Beckwith, S. V. W., Sargent, A. I., Chini, R. S., \& Güsten, R. 1990, AJ, 99, 924

Brasseur, G \& Solomon, S. 1986, in Aeronomy of the Middle Atmosphere, ed. Brasseur, G \& Solomon, S. (D. Reidel Publishing Company)

Farquhar, P. R. A., \& Millar, T. J. 1993, CCP7 Newsletter, 18, 6

Hasegawa, T. I., \& Herbst, E. 1993, MNRAS, 261, 83

Hayashi, C. 1981, Prog. Theor. Phys. Suppl., 70, 35

Millar, T. J., Rawlings, J. M. C., Bennett, A., Brown, P. D. \& Charnley, S. B. 1991, A\&ASS, 87, 585

Nakagawa, Y., Nakazawa, K., \& Hayashi, C. 1981, ICARUS, 45, 517

O'dell, C.R., \& Wen Zheng 1994, ApJ, 436, 194

Prinn, R. G. 1993, in Protostars and Planets III, ed. Levy, E. H., \& Lunine, J. I., (Tucson: Univ. of Arizona Press), 1005

Sandford, S. A., \& Allamandola, L. J. 1993, ApJ, 417, 815

Sargent, A. I., \& Beckwith, S. V. W. 1994, Ap\&SS, 212,181

Strom, K. M., Strom, S. E., Edwards, S, Cabrit, S., and Skrutskie, M. F. 1989, AJ 97, 1451

Umebayashi, T., \& Nakano, T. 1981, PASJ, 33, 617

Williams, D. A. 1993, in Dust and Chemistry in Astronomy, ed. Millar, T. J., \& Williams, D. A. (London: Institute of Physics Publishing), 143

Yamamoto, T. 1991, in Comets in the Post-Halley Era, ed. Newburn, R. L. Jr. et al. (Kluwer Academic Publishers), 361

Yamamoto, T, Nakagawa, N., \& Fukui, Y. 1983, A\&A, 122, 171

This 2-column preprint was prepared with the AAS LATEX 
macros v4.0. 
$\log n_{i} / n_{H}$

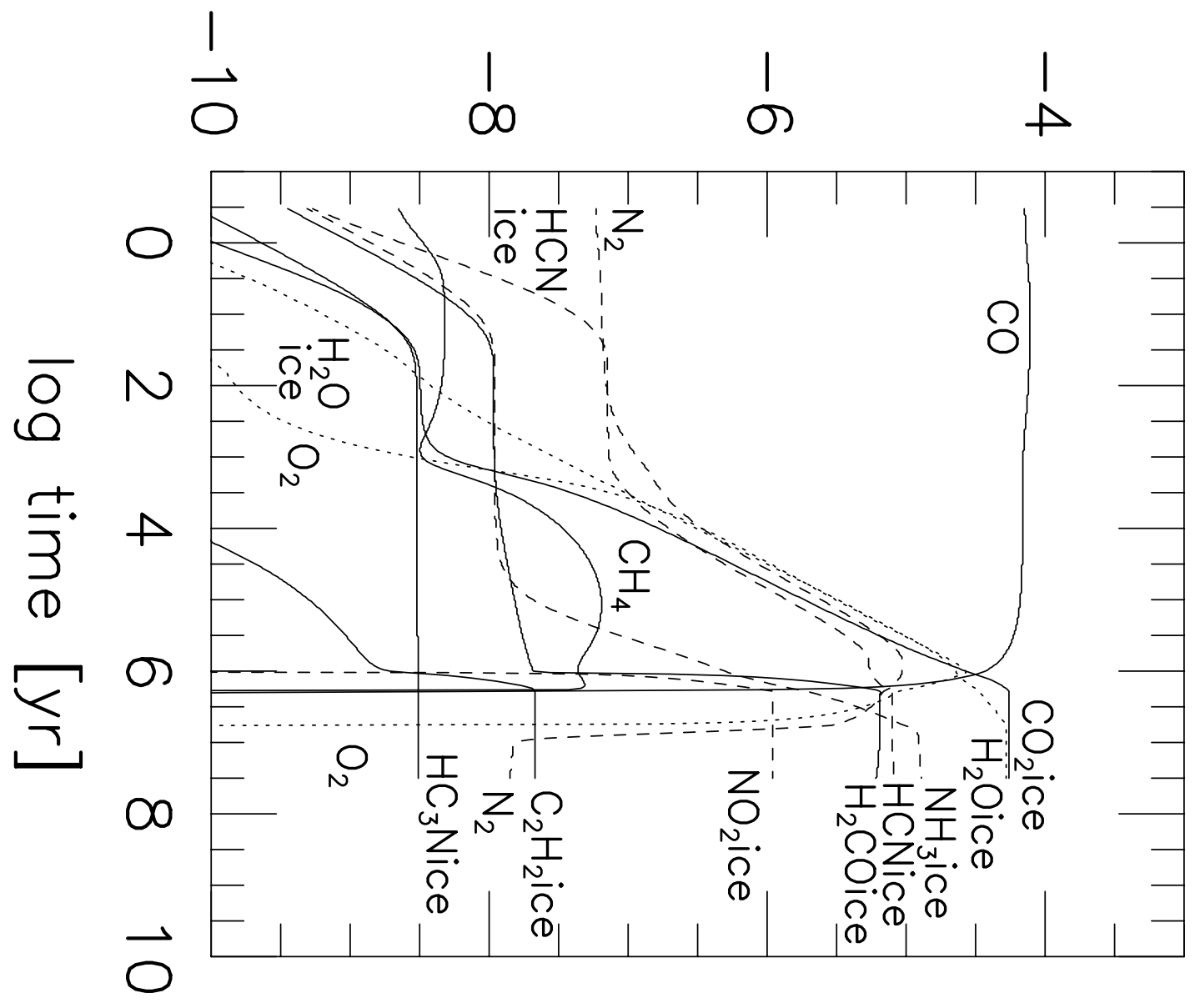


$\log n_{i} / n_{H}$

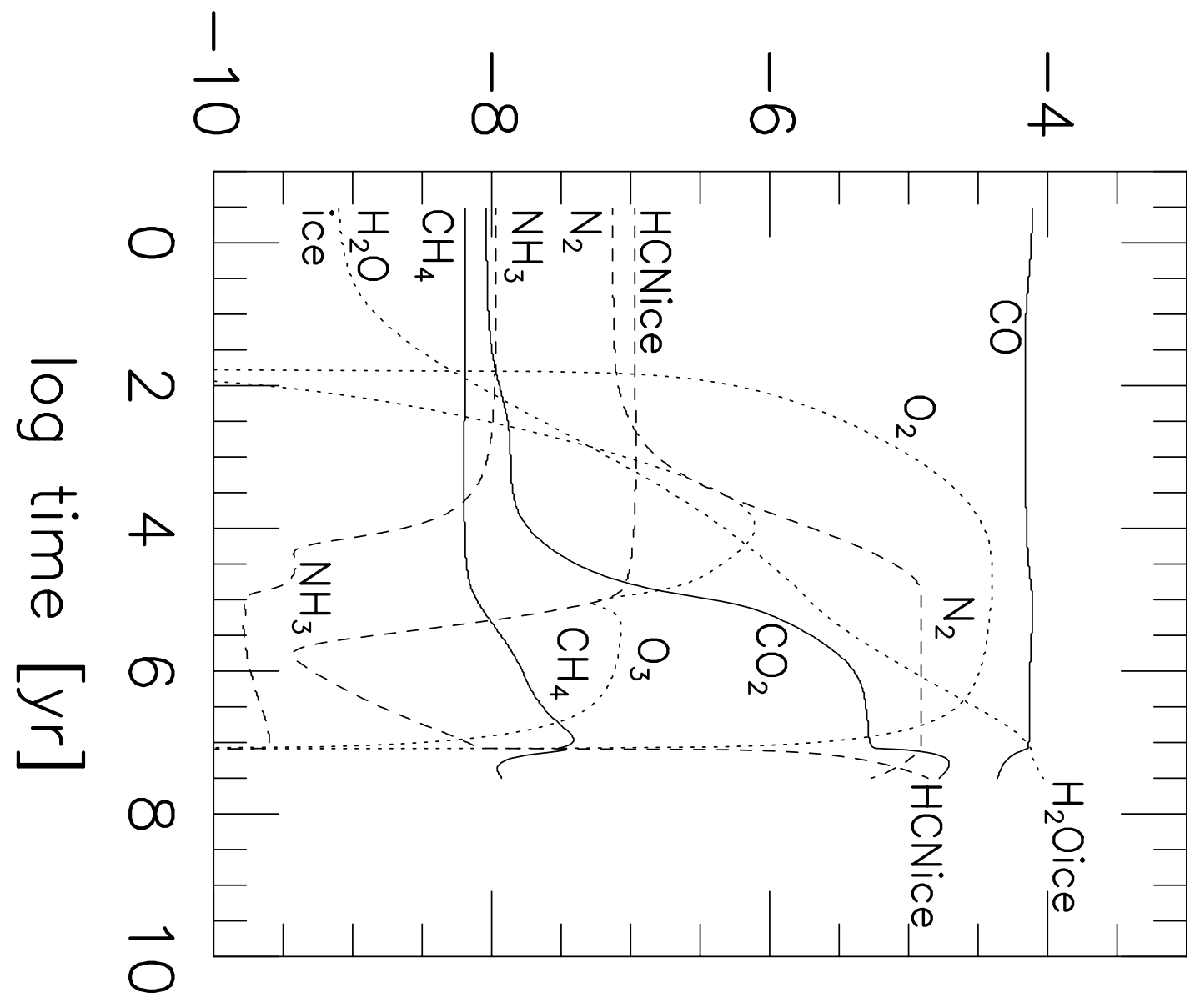


$\log n_{i} / n_{H}$

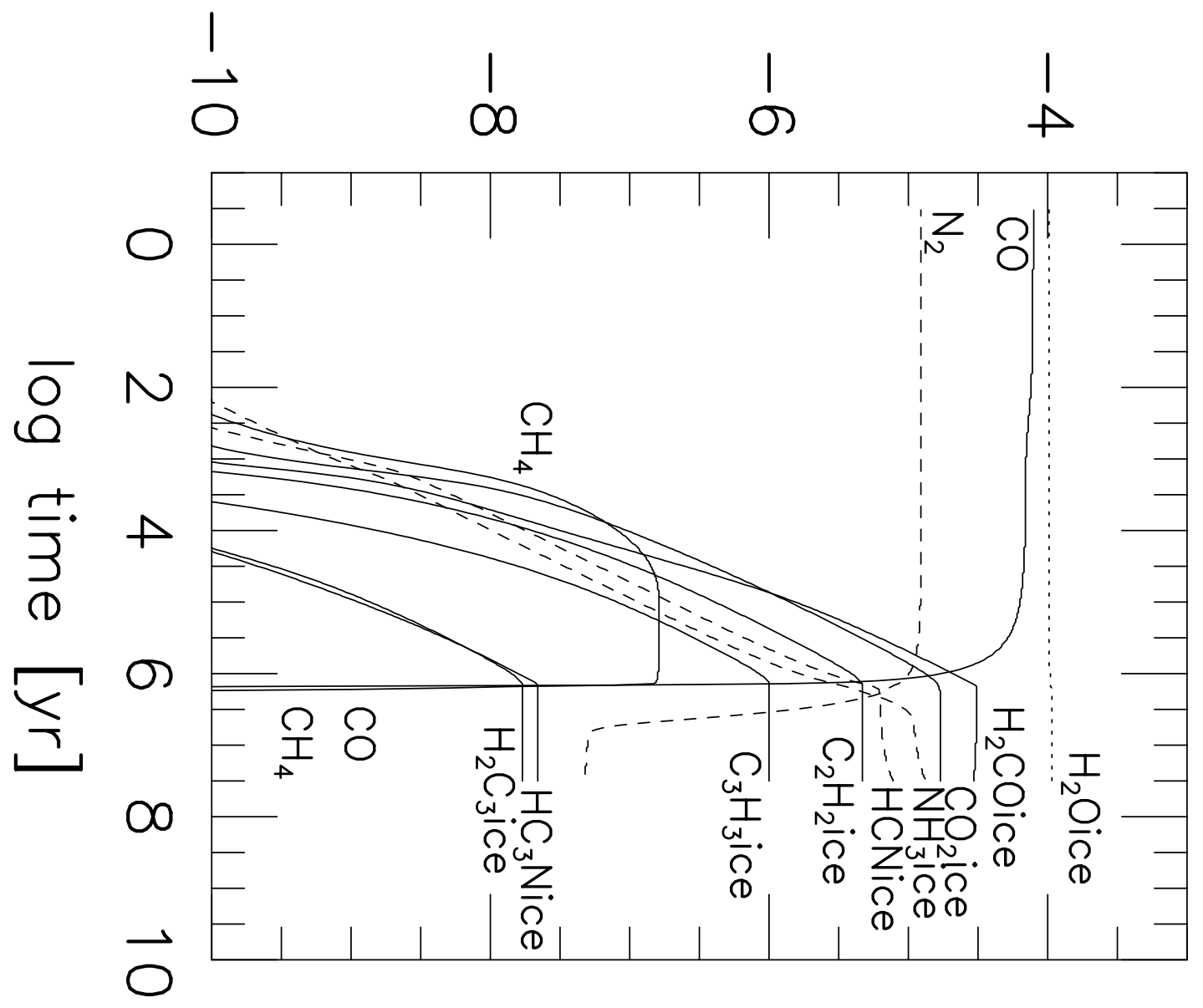

